\title{
AMENABLE REPRESENTATIONS AND COEFFICIENT SUBSPACES OF FOURIER-STIELTJES ALGEBRAS
}

\author{
ROSS STOKKE*
}

\begin{abstract}
Amenable unitary representations of a locally compact group, $G$, are studied in terms of associated coefficient subspaces of the Fourier-Stieltjes algebra $B(G)$, and in terms of the existence of invariant and multiplicative states on associated von Neumann and $C^{*}$-algebras. We introduce Fourier algebras and reduced Fourier-Stieltjes algebras associated to arbitrary representations, and study amenable representations in relation to these algebras.
\end{abstract}

\section{Introduction}

The theory of amenable representations was developed in 1990 by M.E.B. Bekka [3]. Bekka proved analogues of all of the classical invariance properties of amenable groups, including the Følner conditions, and interpreted several amenability theories in terms of amenable representations. In particular, he proved that a locally compact group is amenable if and only if each of its unitary representations is amenable.

We will say that a representation is $H$-amenable if it has almost invariant vectors. We give several characterizations of both amenable and $H$-amenable representations, in terms of associated coefficient subspaces of the FourierStieltjes algebra, and in terms of the existence of certain states upon related von Neumann and $C^{*}$-algebras. These results provide new characterizations of amenable and inner amenable locally compact groups, as well as amenable action on coset spaces. Coefficient subspaces of the Fourier-Stieltjes algebra have been studied by several authors, see for example [1], [4], [6], [7], [9], and [13].

Leptin's theorem [15] states that a locally compact group $G$ is amenable precisely when its Fourier algebra, $A(G)$, has a bounded approximate identity. Leptin's theorem is the starting point to much of the theory connecting amenable locally compact groups to properties of their associated Fourier and group von Neumann algebras. For each representation $\pi$ of $G$, we introduce an associated Fourier algebra $A(\pi)$, and a reduced Fourier-Stieltjes algebra $B(\pi)$.

\footnotetext{
* This research was partly supported by an NSERC grant.

Received October 25, 2004; in revised form December 20, 2004.
} 
In Section 4 we prove that when the Fourier algebra $A(\pi \otimes \bar{\pi})$ has a bounded approximate identity, the representation $\pi$ is necessarily amenable. With regards to the reduced Fourier-Stieltjes algebras, it is shown that the existence of an identity, or a bounded approximate identity, in $B(\pi \otimes \bar{\pi})$ characterizes the amenability of $\pi$. Several examples are provided in Section 5 .

A portion of the material in this paper has appeared in the author's Ph.D. thesis, written under the supervision of Professor Anthony T.-M. Lau at the University of Alberta, (2003). The author would like to express his appreciation to Professor Lau for all of his encouragement and for introducing him to the subject of this work. The author is also very grateful to Professor Fereidoun Ghahramani for his financial support in the spring and summer of 2003. The author thanks the referee for his or her valuable comments which provided significant improvements to this article.

\section{Preliminaries}

Throughout this paper, $G$ is a locally compact group with fixed left Haar measure $d x$ and modular function $\Delta$. References for the material which follows are [1], [5], [8] and [9].

By a representation $\{\pi, \mathscr{C}\}$ of $G$ we will always mean a continuous unitary representation, $\pi$, on a Hilbert space $\mathscr{H}$. A net of unit vectors $\xi_{\alpha}$ in $\mathscr{H}$ is called an almost invariant net of vectors for $\pi$, if

$$
\left\|\pi(s) \xi_{\alpha}-\xi_{\alpha}\right\| \rightarrow 0
$$

uniformly on compact subsets of $G$. The group $G$ endowed with the discrete topology is denoted by $G_{d}$, and $\pi_{d}$ is the representation $\pi$ viewed as a representation of $G_{d}$. If $\xi, \eta \in \mathscr{H}$, then $\xi *_{\pi} \eta(s)=\langle\pi(s) \xi \mid \eta\rangle(s \in G)$ is the associated coefficient function. We write $\pi \cong \gamma$ and $\pi \preceq \gamma$ to respectively indicate unitary equivalence and weak containment.

The Fourier-Stieltjes algebra of $G$ is $B(G)$, and is identified with the dual of the group $C^{*}$-algebra, $C^{*}(G)$. We will be concerned with the $C^{*}$-algebras associated to $\{\pi, \mathscr{H}\}, C_{\pi}^{*}=\pi\left(C^{*}(G)\right)$ and $C_{\delta, \pi}^{*}=C_{\pi_{d}}^{*}$. The von Neumann algebra generated by $\pi$ is the WOT-closure in $B(\mathscr{H})$ of either $\langle\pi(G)\rangle$ or $C_{\pi}^{*}$, (where $\langle\cdot\rangle$ indicates linear span), and is denoted by $V N_{\pi}$. We define $A_{\pi}$, the Fourier space associated to $\pi$, to be the norm-closure in $B(G)$ of $\left\langle\xi *_{\pi} \eta\right.$ : $\xi, \eta \in \mathscr{H}\rangle ; B_{\pi}$, the reduced Fourier-Stieltjes space associated to $\pi$, is the $w^{*}$-closure of $A_{\pi}$ in $B(G)$. The Banach space $A_{\pi}$ may be identified with the predual of $V N_{\pi}$, and $B_{\pi}$ may be identified with the dual of $C_{\pi}^{*}$.

If $\left\{\lambda, L^{2}(G)\right\}$ is the left regular representation of $G, A_{\lambda}=A(G)$ is a closed ideal of $B(G)$, called the Fourier algebra of $G$, and $B_{\lambda}=B_{r}(G)$ is the 
reduced Fourier-Stieltjes algebra of $G$. The reduced group $C^{*}$-algebra of $G$ is $C_{r}^{*}(G)=C_{\lambda}^{*}$, and the group von Neumann algebra of $G$ is $V N(G)=V N_{\lambda}$.

\section{Amenable and $\boldsymbol{H}$-amenable representations}

The following definition of an amenable representation was given by M.E.B. Bekka in [3].

Definition 2.1. A representation $\{\gamma, \mathscr{K}\}$ of $G$ is called amenable if there exists a state $\omega$ on $B(\mathscr{K})$ such that

$$
\omega\left(\gamma(s) x \gamma\left(s^{-1}\right)\right)=\omega(x) \quad(x \in B(\mathscr{K}), s \in G) .
$$

The state $\omega$ is called a $G$-invariant mean for $\gamma$.

For our purposes, we will find it convenient to introduce the following terminology.

Definition 2.2. A representation $\{\pi, \mathscr{H}\}$ will be called $H$-amenable if $\pi$ has an almost invariant net of (unit) vectors. If $\pi_{d}$ is $H$-amenable, then we will say that $\pi$ is $H_{d}$-amenable.

Observe that all statements phrased in terms of $H$-amenability may be interpreted in terms of $H_{d}$-amenability. The following remarks record some elementary properties of $H$-amenable representations.

Remarks 2.3. (1) If $\{\pi, \mathscr{H}\}$ is $H_{d}$-amenable, then it is amenable. To see this, let $\left(\xi_{\alpha}\right)$ be a net of almost invariant vectors for $\pi_{d}$. Consider the vector state $\omega_{\xi_{\alpha}}$ on $B(\mathscr{H})$ defined by $\omega_{\xi_{\alpha}}(T)=\left\langle T \xi_{\alpha} \mid \xi_{\alpha}\right\rangle(T \in B(\mathscr{H}))$, and take $\omega$ to be any $w^{*}$-limit point in $B(\mathscr{H})^{*}$ of $\left(\omega_{\xi_{\alpha}}\right)$. Then it is easily seen that $\omega$ satisfies

$$
\omega(\pi(s) x)=\omega(x \pi(s))=\omega(x) \quad(x \in B(\mathscr{H}), s \in G) .
$$

In particular, $\omega$ is a $G$-invariant mean for $\pi$. Conversely, we note that if there is a state $\omega$ on $B(\mathscr{H})$ such that Equation (1) holds, then $\pi$ is necessarily $H_{d^{-}}$ amenable. This follows, for example, from Proposition 3.1.

(2) The Reiter conditions for amenability and inner amenability, (see for example [18, Proposition 1.13] in conjunction with [16, Exercise 4.4.5]) show that

(i) $G$ is amenable $\Leftrightarrow$ the left regular representation $\left\{\lambda, L^{2}(G)\right\}$ is $H$-amenable;

(ii) $G$ is inner amenable $\Leftrightarrow$ the conjugation representation $\left\{\beta, L^{2}(G)\right\}$ is $H$-amenable.

When $H$ is a closed subgroup of $G$ we similarly obtain that 
(iii) $G$ acts amenably on $G / H \Leftrightarrow$ the quasi-regular representation $\operatorname{Ind} d_{H}^{G} 1_{H}$ is $H$-amenable.

(3) The statements (i), (ii), and (iii) of [3, Remark 1.2] are valid for $H$ amenable representations. Moreover, it is clear that if $\{\pi, \mathscr{H}\}$ contains an $H$ amenable subrepresentation, then $\{\pi, \mathscr{H}\}$ is also $H$-amenable.

The following may be compared to [3, Theorem 1.3].

Proposition 2.4. Let $\left\{\pi_{1}, \mathscr{H}_{1}\right\}, \ldots,\left\{\pi_{n}, \mathscr{H}_{n}\right\}$ be representations of $G$, and let $\pi=\sum_{1}^{n} \oplus \pi_{k}$.If $\pi$ is $H$-amenable, then at least one $\pi_{k}$ is $H$-amenable.

Proof. Let $\mathscr{H}=\sum_{1}^{n} \oplus \mathscr{H}_{k}$, and let $\left(\xi_{\alpha}\right)$ be a net of almost invariant vectors for $\pi$. Write $\xi_{\alpha}=\oplus_{k=1}^{n} \xi_{\alpha, k}$. Then $1=\sum_{k=1}^{n}\left\|\xi_{\alpha, k}\right\|^{2}$ so for some $k$ we may assume, by passing to a subnet if necessary, that for each $\alpha$ we have $\left\|\xi_{\alpha, k}\right\| \geq$ $1 / \sqrt{n}$. The inequality

$$
\left\|\pi(s) \xi_{\alpha}-\xi_{\alpha}\right\| \geq\left\|\pi_{k}(s) \xi_{\alpha, k}-\xi_{\alpha, k}\right\|
$$

now readily yields the $H$-amenability of $\pi_{k}$.

Example 2.5. Let $\mathrm{T}=\{\alpha \in \mathrm{C}:|\alpha|=1\}$ be the circle group and let $\mathscr{H}$ be any Hilbert space. Consider the representation defined by

$$
\iota_{\mathscr{H}}: \mathrm{T} \rightarrow B(\mathscr{H}): \alpha \mapsto \alpha \operatorname{id}_{\mathscr{H}} .
$$

It is obvious that $\iota_{\mathscr{H}}$ is amenable. But, if $\xi$ is any unit vector in $\mathscr{H}$, then $\|\iota \mathscr{H}(-1) \xi-\xi\|=2$ so $\iota \mathscr{H}$ is not $H_{d}$-amenable.

Thus, $H$-amenability is strictly a stronger property than amenability for representations. Moreover, in contrast to the theory of amenable representations (see [3, Theorems 1.3 and 2.2]), even one-dimensional representations can fail to be $H$-amenable, and amenable groups can have representations which are not $H$-amenable. In terms of weak containment, it is well-known that the existence of almost invariant vectors can be simply expressed as follows (see Corollary F.1.5 of [5] and [1, Proposition 3.1]):

Proposition 2.6. The following are equivalent for a representation $\{\pi, \mathscr{H}\}$ of $G$.
(1) $\{\pi, \mathscr{H}\}$ is $H-$ amenable
(2) $1_{G} \preceq \pi$
(3) $1_{G} \in B_{\pi}$

Corollary 5.4 (ii) of [3] states that when $\pi$ and $\gamma$ are amenable representations, the representation $\pi \otimes \gamma$ is also amenable. Observe that if $\Omega$ is a $G$ invariant mean for $\pi \otimes \gamma$ on $B(\mathscr{H} \otimes \mathscr{K})$, then, by defining $\omega(y)=\Omega\left(y \otimes \operatorname{id}_{\mathscr{K}}\right)$ 
$(y \in B(\mathscr{H}))$, we obtain obtain a $G$-invariant mean for $\pi$ on $B(\mathscr{H})$. Thus, we can make the following statement.

Proposition 2.7. Let $\{\pi, \mathscr{H}\}$ and $\{\gamma, \mathscr{K}\}$ be representations of $G$. Then $\pi$ and $\gamma$ are amenable if and only if $\pi \otimes \gamma$ is amenable.

The following is an immediate corollary of [3, Theorem 5.1], Remark 2.3(1), and Proposition 2.7. It shows that all notions of amenability are equivalent for representations of the form $\gamma \otimes \bar{\gamma}$. As well, it shows that any characterization of $\mathrm{H}$-amenable representations yields a characterization of amenable representations and hence, by [3, Theorem 2.2], of amenable locally compact groups.

COROLlary 2.8. Let $\{\gamma, \mathscr{K}\}$ a representation of $G$, and let $\{\bar{\gamma}, \overline{\mathscr{K}}\}$ be its conjugate representation. The following are equivalent.

(1) $\{\gamma, \mathscr{K}\}$ is amenable.

(2) $\{\gamma \otimes \bar{\gamma}, \mathscr{K} \otimes \overline{\mathscr{K}}\}$ is H-amenable.

(3) $\{\gamma \otimes \bar{\gamma}, \mathscr{K} \otimes \overline{\mathscr{K}}\}$ is $H_{d}$-amenable.

(4) $\{\gamma \otimes \bar{\gamma}, \mathscr{K} \otimes \overline{\mathscr{K}}\}$ is amenable.

\section{Invariant means and multiplicative functionals}

We will characterize the amenability and $H$-amenability of a representation $\{\pi, \mathscr{H}\}$ in terms of $A_{\pi}$, and in terms of the existence of certain states on $C_{\delta, \pi}^{*}$, $C_{\pi}^{*}, V N_{\pi}$, and $B(\mathscr{H})$.

Recall that if $\mathscr{A}$ is any Banach algebra, then its dual $\mathscr{A}^{*}$ becomes a Banach $\mathscr{A}$-bimodule through the operations

(2) $\langle\phi \cdot x, y\rangle=\langle\phi, x y\rangle$ and $\langle x \cdot \phi, y\rangle=\langle\phi, y x\rangle \quad\left(\phi \in \mathscr{A}^{*}, x, y \in \mathscr{A}\right)$.

When $\mathscr{A}$ is a $C^{*}$-algebra, the operations in equation (2) are often called Sakaiproducts, and to avoid confusion, we shall write $\phi . x$ and $x . \phi$ for all Sakaiproducts. The predual $\mathscr{M}_{*}$ of a von Neumann algebra $\mathscr{M}$ is always a Banach $\mathscr{M}$-submodule of $\mathscr{M}^{*}$.

According to Proposition 2.8 of [1], for $\mu \in M(G)$, and $u \in A_{\pi}$,

$$
\pi(\mu) . u=u * \Delta \check{\mu} \quad \text { and } \quad u . \pi(\mu)=\check{\mu} * u,
$$

where

$$
\langle h, \check{\mu}\rangle=\int_{G} h\left(s^{-1}\right) d \mu(s) \quad\left(h \in C_{0}(G)\right) .
$$


We will call a state $\phi$ on either $V N_{\pi}$ or $C_{\pi}^{*}$ a $\pi$-topological right invariant mean (or $\pi$-TRIM) if

$$
\phi . \pi(f)=\phi \quad\left(f \in L^{1}(G)_{1}^{+}\right) .
$$

As well, we have left and two-sided versions of this concept: $\pi$-TLIM's and $\pi$-TIM's. A state $\phi$ on $V N_{\pi}$ is a $\pi$-right invariant mean ( $\pi$-RIM) if $\phi . \pi(s)=$ $\phi(s \in G)$. A simple argument shows that any $\pi$-TRIM is a $\pi$-RIM. For $u$ a function on $G$, and $s, t \in G$, we write $l_{s} u(t)=u(s t)$ and $r_{s} u(t)=u(t s)$.

Proposition 3.1. Let $\{\pi, \mathscr{H}\}$ be a representation of $G$. Then the following are equivalent.

(1) $\{\pi, \mathscr{H}\}$ is $H$-amenable.

(2) There is a net $\left(u_{\alpha}\right) \subset A_{\pi} \cap P_{1}(G)$ such that

$$
\left\|l_{s} u_{\alpha}-u_{\alpha}\right\|_{A_{\pi}} \rightarrow 0 \quad\left(\text { and } \quad\left\|r_{s} u_{\alpha}-u_{\alpha}\right\|_{A_{\pi}} \rightarrow 0\right)
$$

uniformly on compact subsets of $G$.

(3) There is a net $\left(u_{\alpha}\right) \subset A_{\pi} \cap P_{1}(G)$ such that

$$
\left\|\mu * u_{\alpha}-u_{\alpha}\right\|_{A_{\pi}} \rightarrow 0 \quad\left(\mu \in M(G)_{1}^{+}\right) .
$$

(4) There is a $\pi$-TRIM ( $\pi$-TIM) $\phi$ on either $V N_{\pi}$ or $C_{\pi}^{*}$.

Proof. (1) $\Rightarrow$ (2) Let $\left(\xi_{\alpha}\right) \subset \mathscr{H}$ be a net of almost invariant vectors for $\pi$. Letting $u_{\alpha}=\xi_{\alpha} *_{\pi} \xi_{\alpha}$, we have $l_{s} u_{\alpha}=\xi_{\alpha} *_{\pi} \pi\left(s^{-1}\right) \xi_{\alpha}$. Thus

$$
\left\|l_{s} u_{\alpha}-u_{\alpha}\right\|_{A_{\pi}}=\left\|\xi_{\alpha} *_{\pi}\left(\pi\left(s^{-1}\right) \xi_{\alpha}-\xi_{\alpha}\right)\right\|_{A_{\pi}} \leq\left\|\pi\left(s^{-1}\right) \xi_{\alpha}-\xi_{\alpha}\right\| \rightarrow 0
$$

uniformly on compact subsets of $G$. The proof of the bracketed part of (2) is similar.

(2) $\Rightarrow$ (3) Observe that $A_{\pi}$ is a Banach $M(G)$-module through Equation (3). It follows from this (see for example [12, Chapter 2]) and Equation (4) that for any $\mu \in M(G)$,

$$
\mu * u=\int_{G} l_{s} u d \check{\mu}(s) \quad \text { (weak integral). }
$$

For $\mu \in M(G)_{1}^{+}$, one can easily establish the inequality

$$
\left\|\mu * u_{\alpha}-u_{\alpha}\right\|_{A_{\pi}} \leq \int_{G}\left\|l_{s} u_{\alpha}-u_{\alpha}\right\|_{A_{\pi}} d \check{\mu}(s) .
$$

Assuming without loss of generality that $\mu \in M(G)_{1}^{+}$has compact support, it is now clear that (3) follows from (2). 
(3) $\Rightarrow(4)$ It is evident that we are assuming that the equation

$$
\left\|u_{\alpha} \cdot \pi(\mu)-u_{\alpha}\right\| \rightarrow 0 \quad\left(\mu \in M(G)_{1}^{+}\right)
$$

holds. Now any $w^{*}$-limit point $\phi$ of $\left(u_{\alpha}\right)$ in $V N_{\pi}^{*}$ is a state satisfying

$$
\phi . \pi(\mu)=\phi \quad\left(\mu \in M(G)_{1}^{+}\right) .
$$

In particular, $\phi$ is a $\pi$-TRIM on $V N_{\pi}$. Setting $\phi_{1}=\left.\phi\right|_{C_{\pi}^{*}}, \phi_{1}$ is positive, $\left\|\phi_{1}\right\| \leq 1$, and $\phi_{1} . \pi(f)=\phi_{1}\left(f \in L^{1}(G)_{1}^{+}\right)$. Moreover, if $f \in L^{1}(G)_{1}^{+}$, then $\|\pi(f)\| \leq 1$ and

$$
\phi_{1}(\pi(f))=\phi(\pi(f))=\phi \cdot \pi(f)(\pi(e))=\phi(\pi(e))=1 .
$$

Thus $\phi_{1}$ is a state on $C_{\pi}^{*}$. To obtain two-sided means, note that if we assume the bracketed part of statement (2), then the net $\left(u_{\alpha}\right)$ found in the proof of (2) $\Rightarrow$ (3) satisfies both equation (5) and

$$
\left\|\pi(\mu) \cdot u_{\alpha}-u_{\alpha}\right\| \rightarrow 0 \quad\left(\mu \in M(G)_{1}^{+}\right) .
$$

Now proceed as above.

(4) $\Rightarrow$ (1) Let $\phi$ be a $\pi$-TRIM on $C_{\pi}^{*}$ and let $\left(e_{\alpha}\right) \subset L^{1}(G)_{1}^{+}$be a bounded approximate identity for $L^{1}(G)$ such that $e_{\alpha}^{*}=e_{\alpha}$. Then $\pi\left(e_{\alpha}\right)$ is a (selfadjoint) bounded approximate identity for $C_{\pi}^{*}$, so for any $f \in L^{1}(G)_{1}^{+}$,

$$
\begin{aligned}
1=\|\phi\| & =\lim \phi\left(\pi\left(e_{\alpha}\right)\right)=\lim \phi \cdot \pi(f)\left(\pi\left(e_{\alpha}\right)\right) \\
& =\lim \phi\left(\pi\left(e_{\alpha}\right) \pi(f)\right)=\phi(\pi(f)) .
\end{aligned}
$$

Thus $\phi \in\left(C_{\pi}^{*}\right)^{*}=B_{\pi}$ satisfies

$$
\int_{G} f(s) d s=\int_{G} f(s) \phi(s) d s \quad\left(f \in L^{1}(G)_{1}^{+}\right) .
$$

Consequently $\phi=1_{G} \in B_{\pi}$, and $\pi$ is $H$-amenable.

Remarks 3.2. (1) The result [3, Corollary 4.5] states that when a representation $\{\pi, \mathscr{H}\}$ is amenable, there exists a net $\left(u_{\alpha}\right) \subset A_{\pi} \cap P_{1}(G)$ such that $\left\|u_{\alpha}^{x}-u_{\alpha}\right\|_{A_{\pi}} \rightarrow 0$ uniformly on compact subsets of $G$ (where $u^{x}(s)=$ $\left.u\left(x^{-1} s x\right)\right)$. Moreover, Bekka proves that the converse holds when $\pi$ is irreducible. Combined with Corollary 2.8, the above result shows that the amenability of a representation $\gamma$ is completely characterized by the existence of a net converging to left or right translation invariance in the Fourier space $A_{\gamma \otimes \bar{\gamma}}$.

(2) Letting $\{\pi, \mathscr{H}\}=\left\{\lambda, L^{2}(G)\right\}$, Proposition 3.1 gives a new characterization of amenability for a locally compact group in terms of its associated group 
von Neumann algebra, and reduced group $C^{*}$-algebra. It is worth noting that the net in condition (2) converges to $1_{G}$ uniformly on compact subsets of $G$. Thus, in this case the nets from (2) are bounded approximate identities in $A(G)$ $\left[10\right.$, Theorem $\left.B_{2}\right]$. Example 5.4 will show that, in general, such a statement is not possible for arbitrary representations.

(3) A representation $\{\pi, \mathscr{H}\}$ is $H$-amenable if and only if there is a net $\left(u_{\alpha}\right) \subset A_{\pi} \cap P_{1}(G)$ such that for every $v \in A(G),\left\|u_{\alpha} v-v\right\| \rightarrow 0$. This follows immediately from the previous remark and $\left[10\right.$, Theorem $\left.B_{2}\right]$.

When our representation is the conjugation representation $\left\{\beta, L^{2}(G)\right\}$, the following contains one of the main results of [20].

COROLlary 3.3. Let $\{\pi, \mathscr{l}\}$ be a representation of $G$. Then the following are equivalent.

(1) $\{\pi, \mathscr{H}\}$ is $H$-amenable.

(2) There is state $\phi$ on $V N_{\pi}$ (or $\left.B(\mathscr{H})\right)$ such that $\phi(\pi(\mu))=\mu(G),(\mu \in$ $M(G)$ ).

(3) There is state $\phi$ on $C_{\pi}^{*}\left(\right.$ or $V N_{\pi}$ or $\left.B(\mathscr{H})\right)$ such that $\phi(\pi(f))=$ $\int_{G} f(s) d s,\left(f \in L^{1}(G)\right)$.

Proof. If $\pi$ is $H$-amenable, then the proof of Proposition 3.1 yields a state $\phi$ on $V N_{\pi}$ satisfying Equation (6). Then

$$
\int_{G} d \mu(s)=1=\phi(\pi(e))=\phi(\pi(\mu)) \quad \mu \in M(G)_{1}^{+} .
$$

Statement (2) now follows by considering the Jordan decomposition of an arbitrary measure $\mu \in M(G)$. That statement (2) implies statement (3) is obvious, and it is clear that statement (3) implies that $\phi=1_{G} \in B_{\pi}$.

The following theorem generalizes and extends [2, Theorems 1 and 1'] due to E. Bédos in the case of the left regular representation. The proof is different from the one in [2].

THEOREM 3.4. The following are equivalent for a representation $\{\gamma, \mathscr{K}\}$ of $G$.

(1) $\{\gamma, \mathscr{K}\}$ is amenable.

(2) There is a non-zero multiplicative linear functional on $C_{\gamma \otimes \bar{\gamma}}^{*}$.

(3) There is a non-zero multiplicative linear functional on $C_{\delta, \gamma \otimes \bar{\gamma}}^{*}$.

(4) There is a continuous character on $G$ which is weakly contained in $\gamma \otimes \bar{\gamma}$ $\left[\right.$ or $\left.(\gamma \otimes \bar{\gamma})_{d}\right]$.

(5) There is a finite dimensional representation on $G$ which is weakly contained in $\gamma \otimes \bar{\gamma}\left[o r(\gamma \otimes \bar{\gamma})_{d}\right]$. 
Proof. We will exhibit the equivalence of statements (1), (2), and the nonbracketed parts of (4) and (5). Observe that amenability of $\gamma$ and $\gamma_{d}$ are equivalent, and that $\gamma_{d} \otimes \overline{\gamma_{d}}=(\gamma \otimes \bar{\gamma})_{d}$. By definition, $C_{\delta, \gamma \otimes \bar{\gamma}}^{*}=C_{(\gamma \otimes \bar{\gamma})_{d}}^{*}$, so the equivalence of all remaining statements will follow.

$(1) \Rightarrow(2)$ The representation $\gamma$ is amenable, so $1_{G} \in B_{\gamma \otimes \bar{\gamma}}$, which is a non-zero multiplicative linear functional on $C_{\gamma \otimes \bar{\gamma}}^{*}$.

(2) $\Rightarrow$ (4) Let $\sigma \in B_{\gamma \otimes \bar{\gamma}}$ be a non-zero multiplicative linear functional on $C_{\gamma \otimes \bar{\gamma}}^{*}$. Then $\sigma$ is necessarily a state on $C_{\gamma \otimes \bar{\gamma}}^{*}$, which is to say that

$$
\sigma \in B_{\gamma \otimes \bar{\gamma}} \cap P_{1}(G)=\left\{u \in P(G): u(e)=1 \text { and } \pi_{u} \preceq \gamma \otimes \bar{\gamma}\right\},
$$

(see [1] and [8]). If we view $\sigma$ as an element of $L^{\infty}(G)$, it is evident that $\sigma$ is a $*$-representation of $L^{1}(G)$ into $C$. Hence, $\sigma$ is a character on $G$ and it follows that $\sigma=\pi_{\sigma}$. Thus, $\sigma \preceq \gamma \otimes \bar{\gamma}$.

(4) $\Rightarrow(5)$ is trivial.

(5) $\Rightarrow$ (1) By [3, Theorem 1.3(i)], finite-dimensional representations are amenable, so it follows from [3, Corollary 5.3] that $\gamma \otimes \bar{\gamma}$ is amenable. Now $\gamma$ is amenable by Corollary 2.8 .

REMARKs 3.5. (1) Note that the left regular representation $\lambda$ is quasiequivalent to $\lambda \otimes \bar{\lambda}$, and consequently $\lambda_{d}$ is quasi-equivalent to $(\lambda \otimes \bar{\lambda})_{d}$. It follows that there are $*$-isomorphisms between $C_{\lambda \otimes \bar{\lambda}}^{*}$ and $C_{r}^{*}(G)$, and between $C_{\delta, \lambda \otimes \bar{\lambda}}^{*}$ and $C_{\delta, \lambda}^{*}$. In particular, [2, Theorem 1] is an immediate consequence of Theorem 3.4.

(2) The continuous character $\iota=\iota \mathscr{H}$ from Example 2.5, is not $H$-amenable. Nevertheless $C_{\iota}^{*}$ has a non-zero multiplicative linear functional, (and indeed each of the statements (2)-(5) holds when $\iota$ replaces $\gamma \otimes \bar{\gamma}$ ).

(3) Theorem 6.8 of [3], (combined with Corollary 2.8), says that if $C_{\delta, \gamma \otimes \bar{\gamma}}^{*}$ has a central state, and if $C_{\delta, \gamma \otimes \bar{\gamma}}^{*}$ is amenable, then $\gamma$ is amenable. Theorem 3.4 may thus be seen as a partial converse to this statement.

We turn now to an examination of the above statements with weak containment replaced by containment. For a given representation $\{\gamma, \mathscr{K}\}$, put $t(\gamma)=\gamma \otimes \bar{\gamma}$. With this notation, Corollary 2.8 says $1_{G} \preceq t(\gamma)$ if and only if $1_{G} \preceq t(t(\gamma))$.

If $\gamma$ is quasi-equivalent to a subrepresentation of $\pi$, we will write $\gamma \preceq Q \pi$ and when $\gamma$ is contained in $\pi$, we write $\gamma \subset \pi$. It is well-known that $1_{G} \subset$ $t(\gamma)$ if and only if $\gamma$ contains a finite-dimensional subrepresentation, (see for example [5, Proposition A.1.10]). We note as well that tensor products preserve quasi-equivalence.

Proposition 3.6. Let $\{\gamma, \mathscr{K}\}$ be a representation of $G$. The following are equivalent. 
(1) $V N_{\gamma \otimes \bar{\gamma}}$ has a non-zero normal multiplicative linear functional.

(2) $A_{\gamma \otimes \bar{\gamma}}$ contains a continuous character.

(3) $1_{G} \subset t(t(\gamma))$.

(4) $1_{G} \subset t(\gamma)=\gamma \otimes \bar{\gamma}$.

Proof. (1) $\Rightarrow$ (2) Let $\sigma \in A_{\gamma \otimes \bar{\gamma}}$ be a nonzero multiplicative linear functional on $V N_{\gamma \otimes \bar{\gamma}}$. We have $\sigma(s)=\langle(\gamma \otimes \bar{\gamma})(s), \sigma\rangle$, so $\sigma(s t)=\sigma(s) \sigma(t)$, $(s, t \in G)$. Moreover $\sigma$ is a state on $V N_{\gamma \otimes \bar{\gamma}}$, so $\sigma\left(s^{-1}\right)=\left\langle\gamma \otimes \bar{\gamma}(s)^{*}, \sigma\right\rangle=$ $\overline{\langle\gamma \otimes \bar{\gamma}(s), \sigma\rangle}=\overline{\sigma(s)}$, and we have $1=\sigma(e)=\sigma\left(s s^{-1}\right)=|\sigma(s)|^{2}$. Thus $\sigma$ is a character on $G$.

(2) $\Rightarrow$ (3) Let $\sigma \in A_{\gamma \otimes \bar{\gamma}}$ be a continuous character on $G$. Then $A_{\sigma} \subset A_{\gamma \otimes \bar{\gamma}}$, so by [1, Corollaire 3.14], $\sigma \preceq_{Q} \gamma \otimes \bar{\gamma}$. Therefore $1_{G} \cong \sigma \otimes \bar{\sigma} \preceq_{Q}(\gamma \otimes$ $\bar{\gamma}) \otimes \overline{(\gamma \otimes \bar{\gamma})}=t(t(\gamma))$, from which it follows that $1_{G} \subset t(t(\gamma))$.

(3) $\Rightarrow$ (4) If $1_{G} \subset t(t(\gamma))$, then, as noted above, $t(\gamma)$ contains a finitedimensional subrepresentation. From the comments at the top of page 386 of [3], there is a normal $G$-invariant mean $\Omega$ for $\gamma \otimes \bar{\gamma}$ on $B(\mathscr{K} \otimes \overline{\mathscr{K}})$. The map $y \mapsto y \otimes i d_{\overline{\mathscr{K}}}: B(\mathscr{K}) \rightarrow B(\mathscr{K} \otimes \overline{\mathscr{K}})$ is $\sigma-\sigma$ continuous, so we can obtain a normal $G$-invariant mean $\omega$ for $\gamma$, (see the paragraph preceding Proposition 2.7). By [3, Theorem 1.4], $\gamma$ contains a finite-dimensional subrepresentation, and hence $1_{G} \subset \gamma \otimes \bar{\gamma}$.

(4) $\Rightarrow$ (1) If $1_{G} \subset \gamma \otimes \bar{\gamma}$, then $1_{G} \in A_{\gamma \otimes \bar{\gamma}}$. Now $1_{G}$ is multiplicative on $\langle(\gamma \otimes \bar{\gamma})(G)\rangle$ which is $\sigma$-dense in $V N_{\gamma \otimes \bar{\gamma}}$. That $1_{G}$ is a normal multiplicative linear functional on $V N_{\gamma \otimes \bar{\gamma}}$ now follows from separate $\sigma$-continuity of multiplication in $V N_{\gamma \otimes \bar{\gamma}}$.

REMARKS 3.7. (1) Given a representation $\pi$, one can also prove the following equivalence:

$V N_{\pi}$ has a normal $\pi$-TRIM (or $\pi$-RIM) $\Leftrightarrow 1_{G} \in A_{\pi} \Leftrightarrow 1_{G} \subset \pi$

(2) Corollary 5.9 of [3] shows that for groups $G$ with Khazdan's property (T), a representation $\gamma$ is amenable if and only if $1_{G} \subset \gamma \otimes \bar{\gamma}$. Thus Proposition 3.6 (and also the previous remark) characterizes the amenable representations of groups with property $(\mathrm{T})$.

\section{The Fourier algebras $A(\pi)$}

In the preliminary section we defined the Fourier spaces $A_{\pi}$ and noted that $A_{\lambda}$ is the Fourier algebra $A(G)$. It is however quite rare for a Fourier space $A_{\pi}$ to be an algebra [1, Proposition 3.26]. In this section we will define and study an analogue of the Fourier algebra for an arbitrary representation. Leptin's theorem [15] says that a locally compact group $G$ is amenable if and only 
if the Fourier algebra $A(G)$ has a bounded approximate identity. In terms of representations this says that $(H-)$ amenability of the left regular representation $\left\{\lambda, L^{2}(G)\right\}$ is characterized by the existence of a bounded approximate identity in $A_{\lambda}$. The main result of this section is one direction of Leptin's theorem for amenable representations.

Let $A(\pi)$ denote the closed subalgebra of $B(G)$ generated by the coefficients $\xi *_{\pi} \eta$ of $\pi$. We will refer to $A(\pi)$ as the Fourier algebra associated to $\pi$. It is not difficult to see that $A(\pi)$ is closed under left and right translations, so by [1, Théorème 3.17] $A(\pi)=A_{\tau_{\pi}}$ for some representation $\tau_{\pi}$ of $G$. We begin with an explicit description of this representation which we will find useful.

Let $\{\pi, \mathscr{H}\}$ be a representation of $G$. For a positive integer $n$ we employ the notation

$$
\pi^{\otimes n}=\otimes_{i=1}^{n} \pi \quad \text { and } \quad \mathscr{H}^{\otimes n}=\otimes_{i=1}^{n} \mathscr{H} .
$$

Lemma 4.1. Let $\{\pi, \mathscr{H}\}$ be a representation of $G$ and consider the associated representation

$$
\left\{\tau_{\pi}, \mathscr{H}_{\tau_{\pi}}\right\}=\left\{\sum_{n=1}^{\infty} \oplus\left(\pi^{\otimes n}\right), \sum_{n=1}^{\infty} \oplus\left(\mathscr{H}^{\otimes n}\right)\right\} .
$$

Then $A(\pi)=A_{\tau_{\pi}}=A\left(\tau_{\pi}\right)$.

Proof. In fact, this follows from results in [1]. First note that by [1, Théorème 3.9], every $u \in A_{\tau_{\pi}}$ may be written as a sum $u=\sum_{n=1}^{\infty} u_{n}$ where $u_{n} \in A_{\pi^{\otimes n}}$ and $\sum_{n=1}^{\infty}\left\|u_{n}\right\|<\infty$. It follows from [1, Proposition 3.25] that $A_{\pi \otimes n} \subset A(\pi)$ and so $A_{\tau_{\pi}} \subset A(\pi)$. For the reverse containment, we need to show that $A_{\tau_{\pi}}$ is itself an algebra. By [1, Proposition 3.26] this is so if (and only if) $\tau_{\pi} \otimes \tau_{\pi}$ is quasi-equivalent to a subrepresentation of $\tau_{\pi}$. But this certainly is the case: For example, it is easily checked that $\infty\left(\tau_{\pi} \otimes \tau_{\pi}\right) \cong \infty\left(\sum_{n=2}^{\infty} \oplus \pi^{\otimes n}\right)$.

In [14] A.T.-M. Lau calls a Banach algebra $A$ an $F$-algebra if $A$ is the predual of some von Neumann algebra $\mathscr{M}$ such that the identity element of $\mathscr{M}$ is a multiplicative linear functional on $A$. We remark that $F$-algebras are now commonly called Lau algebras and we shall refer to them as such. Observe that if $\left\{\tau, \mathscr{H}_{\tau}\right\}$ is a representation of $G$ for which $A_{\tau}$ is an algebra, then $\tau(e)$ is the identity in $V N_{\tau}=A_{\tau}^{*}$ and $\langle u, \tau(e)\rangle=u(e)\left(u \in A_{\tau}\right)$. It follows that $A_{\tau}$ is a Lau algebra. Thus, for any representation $\{\pi, \mathscr{H}\}$ of $G, A(\pi)$ is a Lau algebra.

Lemma 4.2. Let $\{\pi, \mathscr{H}\}$ be a representation of $G$. Then $\{\pi, \mathscr{H}\}$ is amenable if and only if $\left\{\tau_{\pi}, \mathscr{H}_{\tau_{\pi}}\right\}$ is amenable. 
Before we prove this, we remark that it has been suggested to the author that if [3, Theorem 1.3(iii)] carried over to infinite sums, then Lemma 4.2 would follow from Proposition 2.7. We do not know if such an infinite version of [3, Theorem 1.3(iii)] is true or not, however we take this opportunity to observe that the proof (at least) which is given in [3] does not work in the infinite case. To see this, note that if $\mathscr{H}=\ell^{2}$, then $\iota_{\mathscr{H}}=\sum_{n=1}^{\infty} \oplus \iota_{\mathscr{H}}$, where $\iota \mathscr{H}$ is as defined in Example 2.5 and each $\mathscr{H}_{n}=\mathrm{C}$. Now let $\left(\omega_{n}\right)$ be the sequence of vector states associated to the standard basis of $\ell^{2}$, and let $\widetilde{M}$ be a $w^{*}$-limit point of $\left(\omega_{n}\right)$. Then $\widetilde{M}$ is a $G$-invariant mean for $\iota_{\mathscr{C}}$, but it is readily verified that $\widetilde{M}\left(P_{n}\right)=0$ for each projection $P_{n}$ onto $\mathscr{H}_{n}$. For this reason, we give the following proof of Lemma 4.2. (Observe that, just as proved below, $\tau_{\pi}$ is $H$-amenable whenever $\pi$ is $H$-amenable. It will be shown in Section 5 that the converse is not true, so there is no infinite version of Proposition 2.4).

Proof. Suppose first that $\pi$ is amenable. Then $\tau_{\pi}=\sum_{n=1}^{\infty} \oplus\left(\pi^{\otimes n}\right)$ has an amenable subrepresentation and it follows from [3, Theorem 1.3(ii)] that $\tau_{\pi}$ is amenable. For the converse, suppose that $\omega$ is a state on $B\left(\mathscr{H}_{\tau_{\pi}}\right)$ such that

$$
\omega\left(\tau_{\pi}(s) B \tau_{\pi}\left(s^{-1}\right)\right)=\omega(B) \quad\left(s \in G, \quad B \in B\left(\mathscr{H}_{\tau_{\pi}}\right) .\right.
$$

Define

$$
\Lambda: B(\mathscr{H}) \rightarrow B\left(\mathscr{H}_{\tau_{\pi}}\right): B \mapsto \sum_{n=1}^{\infty} \oplus\left(B \otimes\left(\otimes_{k=2}^{n} \operatorname{id}_{\mathscr{H}}\right)\right) .
$$

Then it is easy to see that $\Lambda$ is a linear isometry such that $\Lambda\left(\mathrm{id}_{\mathscr{C}}\right)=\mathrm{id}_{\mathscr{\ell}_{\tau_{\pi}}}$, and, for any $B \in B(\mathscr{H})$ and $s \in G, \tau_{\pi}(s) \Lambda(B) \tau_{\pi}\left(s^{-1}\right)=\Lambda\left(\pi(s) B \pi\left(s^{-1}\right)\right)$. Therefore, if we define

$$
m(B)=\omega(\Lambda(B)) \quad(B \in B(\mathscr{H})),
$$

then it is clear that $m$ is a $G$-invariant mean on $B(\mathscr{H})$ for $\{\pi, \mathscr{H}\}$.

Let $\{\pi, \mathscr{H}\}$ be a representation of $G$. We define the reduced Fourier-Stieltjes algebra, $B(\pi)$, associated to $\pi$ as the weak*-closure of $A(\pi)$ in $B(G)$. That is, $B(\pi)=B_{\tau_{\pi}}$. By [1, Proposition 2.20], an element $u \in B(G)$ belongs to $B_{\tau_{\pi}}$ precisely when there is a bounded net in $A_{\tau_{\pi}}=A(\pi)$ which converges to $u$ uniformly on compact subsets of $G$. From this it follows easily that $B(\pi)$ is indeed a Banach subalgebra of $B(G)$. When our representation is the left regular representation $\left\{\lambda, L^{2}(G)\right\}$, we have $B(\lambda)=B_{\lambda}=B_{r}(G)$, the reduced Fourier-Stieltjes algebra of $G$.

If $\{\pi, \mathscr{H}\}$ is a representation such that $A_{\pi}=A(\pi)$, let $V N_{\pi}=A_{\pi}^{*}$ have its canonical dual $A_{\pi}$-module structure. 
Lemma 4.3. Let $\{\pi, \mathscr{H}\}$ be a representation of $G$ such that $A_{\pi}=A(\pi)$.

(1) The module action of $A_{\pi}$ on $V N_{\pi}$ restricted to $\pi\left(L^{1}(G)\right)$ is given by pointwise multiplication. That is,

$$
u \cdot \pi(f)=\pi(u f) \quad\left(u \in A_{\pi}, f \in L^{1}(G)\right) .
$$

Consequently $C_{\pi}^{*}$ is a closed $A_{\pi}$-submodule of $V N_{\pi}$.

(2) The dual module action of $A_{\pi}$ on $\left(C_{\pi}^{*}\right)^{*}=B_{\pi}$ is given by pointwise multiplication. That is,

$$
u \cdot \phi=u \phi \quad\left(u \in A_{\pi}, \phi \in B_{\pi}\right)
$$

(3) If $A(\pi)$ has a bai, then $\{\pi, \mathscr{H}\}$ is $H$-amenable.

Proof. (1) For $u, v \in A_{\pi}$ and $f \in L^{1}(G)$ we have

$$
\langle v, u \cdot \pi(f)\rangle=\langle v u, \pi(f)\rangle=\int_{G} v(s) u(s) f(s) d s=\langle v, \pi(u f)\rangle .
$$

As $C_{\pi}^{*}=\overline{\pi\left(L^{1}(G)\right)} \|^{\|\cdot\|_{B(\mathscr{C})}}$ it follows that $C_{\pi}^{*}$ is an $A_{\pi}$-submodule of $V N_{\pi}$.

(2) Let $u \in A_{\pi}, \phi \in\left(C_{\pi}^{*}\right)^{*}=B_{\pi}$. Then $u \phi \in B_{\pi}$ and for any $f \in L^{1}(G)$,

$$
\begin{aligned}
\langle\pi(f), u \cdot \phi\rangle & =\langle\pi(f) \cdot u, \phi\rangle=\langle\pi(f u), \phi\rangle \\
& =\int_{G} f(s) u(s) \phi(s) d s=\langle\pi(f), u \phi\rangle,
\end{aligned}
$$

where we have used part (1).

(3) Suppose that $A(\pi)=A_{\pi}$ has a bai $\left(e_{\alpha}\right)$ and let $\phi$ be a $w^{*}$-limit point of $\left(e_{\alpha}\right)$ in $V N_{\pi}^{*}$; assume without loss of generality that $e_{\alpha} \rightarrow \phi w^{*}$. Then for any $u \in A_{\pi}$ and $x \in V N_{\pi}$ we have

$$
\langle x, u \cdot \phi\rangle=\langle x \cdot u, \phi\rangle=\lim \left\langle x \cdot u, e_{\alpha}\right\rangle=\lim \left\langle x, u e_{\alpha}\right\rangle=\langle x, u\rangle .
$$

Thus $u \cdot \phi=u\left(u \in A_{\pi}\right)$. Letting $\phi_{1} \in\left(C_{\pi}^{*}\right)^{*}=B_{\pi}$ denote the restriction of $\phi$ to $C_{\pi}^{*}$, part (2) of this lemma gives

$$
u=u \cdot \phi_{1}=u \phi_{1} \quad\left(u \in A_{\pi}\right)
$$

Suppose that $\xi$ is any unit vector in $\mathscr{H}$. Then given any $s \in G$, letting $\eta=\pi(s) \xi$ and $u=\xi *_{\pi} \eta$, we have

$$
1=u(s)=u(s) \phi_{1}(s)=\phi_{1}(s) .
$$

Thus $\phi_{1}=1_{G} \in B_{\pi}$ and by Proposition $2.6\{\pi, \mathscr{H}\}$ is $H$-amenable. 
THEOREM 4.4. Let $\{\gamma, \mathscr{K}\}$ be a representation of $G$. If either $A(\gamma)$ or $A(\gamma \otimes \bar{\gamma})$ has a bounded approximate identity, then $\gamma$ is amenable.

Proof. If $A(\gamma)$ has a bounded approximate identity, then by Lemma 4.1 and Lemma 4.3, $\tau_{\gamma}$ is $H$-amenable and therefore amenable. The amenability of $\gamma$ now follows from Lemma 4.2. If $A(\gamma \otimes \bar{\gamma})$ has a bai, then the above argument and Corollary 2.8 show that $\gamma$ is amenable.

In the next section we will give an example of an $H$-amenable representation $\pi$ for which $A(\pi)=A_{\pi}$ fails to have an approximate identity. Unfortunately, we have been unable to determine whether the $(H$-)amenability of $\gamma$ guarantees the existence of a bai in $A(\gamma \otimes \bar{\gamma})$. Observe, however, that $\lambda \otimes \bar{\lambda}$ and $\lambda$ are quasi-equivalent, so $A(G)=A(\lambda)=A(\lambda \otimes \bar{\lambda})$. For reduced Fourier-Stieltjes algebras we are able to say more.

THEOREM 4.5. The following are equivalent for a representation $\{\gamma, \mathscr{K}\}$ of $G$.

(1) $\{\gamma, \mathscr{K}\}$ is amenable.

(2) The reduced Fourier-Stieltjes algebra $B(\gamma \otimes \bar{\gamma})$ has an identity.

(3) The reduced Fourier-Stieltjes algebra $B(\gamma \otimes \bar{\gamma})$ has a bounded approximate identity.

Proof. (1) $\Rightarrow$ (2) This is an immediate consequence of [3, Theorem 5.1] and [1, Proposition 3.1] (quoted in this article respectively as Corollary 2.8 and Proposition 2.6).

(2) $\Rightarrow(3)$ is trivial.

(3) $\Rightarrow$ (1) Suppose that $B(\gamma \otimes \bar{\gamma})$ has a bounded approximate identity. It follows from Proposition 2.24 of [1] that $B(\gamma \otimes \bar{\gamma})=B_{\tau_{\gamma \otimes \bar{\gamma}}}=A_{\pi}=A(\pi)$, for some representation $\pi$ of $G$. Now $A_{\pi}$ is $w^{*}$-closed, and by Lemma 4.3 $\pi$ is $H$-amenable, so $1_{G} \in B_{\pi}=A_{\pi}$. Thus $1_{G} \in B_{\tau_{\gamma \otimes \bar{\gamma}}}$ and it follows that $\tau_{\gamma \otimes \bar{\gamma}}$ is $H$-amenable. That $\gamma$ is amenable now follows as it did in the proof of Theorem 4.4.

As noted earlier, $A(\pi)$ is a commutative Lau algebra. It follows directly from [14, Example(1) Page 168] and [14, Theorem 4.10] that $A(\pi)$ has a bai precisely when the closed ideal $I_{\pi}=\{u \in A(\pi): u(e)=0\}$ of $A(\pi)$ has a bai.

Proposition 4.6. Let $G$ be a locally compact group. Then $G$ is amenable if and only if $B_{r}(G)$ contains a non-trivial translation-invariant closed subalgebra with a bounded approximate identity.

Proof. The forward implication follows from Leptin's theorem. For the converse, note that any such subalgebra is of the form $A_{\pi}=A(\pi)$ with $\pi \preceq \lambda$ 
[1, Proposition 3.1 and Théorème 3.17]. The statement now follows from Lemma 4.3(3), Remark 2.3(2) and Proposition 2.6.

\section{Examples}

The equivalence of $H$ and $H_{d}$-amenability for representations of the form $\gamma \otimes \bar{\gamma}$ is really just Reiter's condition for amenable representations (see Theorems 4.3 and 5.1 of [3]). Moreover, for each of the representations discussed in Remark 2.3(2), it is known that $H_{d}$-amenability implies $H$-amenability. (A generalized approach which simultaneously yields Reiter's condition in all of the above cases may be found in [18, Section 1]). It is natural to wonder whether Reiter's condition holds in general for $H$-amenability, that is, whether $H_{d}$-amenability always implies $H$-amenability. Example 5.2 will show that this is not the case. If, however, $\{\pi, \mathscr{H}\}$ is continuous with respect to the norm topology on $B(\mathscr{H})$, then a standard compactness argument shows that $\pi$ is $H$ amenable, whenever it is $H_{d}$-amenable. This last statement may also be derived from results in [17], where norm continuous representations are studied.

We first show that every infinite, compact, abelian group has an $H_{d}$-amenable representation which is not $H$-amenable. This is a consequence of two facts which are summarized in the following remarks.

ReMARKs 5.1. (i) Let $G$ be an infinite compact abelian group, and let $\Gamma$ be a subset of the dual group of $G, \widehat{G}$, such that $\widehat{G}=\Gamma \cup \Gamma^{-1} \cup\left\{1_{G}\right\}$. Then $1_{G_{d}}$ is weakly contained in $\Gamma_{d}=\left\{\gamma_{d}: \gamma \in \Gamma\right\}$. To see this, notice that $\widehat{G}$ is dense $\widehat{G_{d}}\left[11\right.$, Corollary 26.16] and, because $G$ is infinite, $\widehat{G_{d}}$ is not discrete. Therefore, $1_{G_{d}}$ belongs to the closure in $\widehat{G_{d}}$ of $\Gamma \cup \Gamma^{-1}$. Hence there is a net $\left(\gamma_{\alpha}\right) \subset \Gamma \cup \Gamma^{-1}$ converging to 1 on finite subsets of $G$. This clearly implies the existence of such a net in $\Gamma$, and thus, $1_{G_{d}}$ is weakly contained in $\Gamma_{d}$.

(ii) Let $G$ be any compact group, and let $\{\pi, \mathscr{H}\}$ be a representation of $G$ such that $\pi$ does not contain $1_{G}$. Then $\pi$ does not weakly contain $1_{G}$. To see this, first note that the orthogonality relations for compact groups, (see for example [11, Theorems 27.15 and 27.44]) show that

$$
\int_{G}\langle\pi(s) \xi \mid \eta\rangle d s=0 \quad(\xi, \eta \in \mathscr{H}) .
$$

Suppose that $1_{G} \preceq \pi$. Then there is a net $\left(\xi_{\alpha}\right)$ of unit vectors in $\mathscr{H}$ such that $\sup _{s \in G}\left\|\pi(s) \xi_{\alpha}-\xi_{\alpha}\right\| \rightarrow 0$. But $\left\|\pi(s) \xi_{\alpha}-\xi_{\alpha}\right\|^{2}=2\left(1-\operatorname{Re}\left\langle\pi(s) \xi_{\alpha} \mid \xi_{\alpha}\right\rangle\right)$, so

$$
2=2\left(1-\operatorname{Re} \int_{G}\left\langle\pi(s) \xi_{\alpha} \mid \xi_{\alpha}\right\rangle d s\right) \leq \sup _{s \in G}\left\|\pi(s) \xi_{\alpha}-\xi_{\alpha}\right\|^{2},
$$

which yields a contradiction. 
EXAMPLE 5.2. Let $G$ be any infinite, compact, abelian group, and let $\Gamma$ be a subset of $\widehat{G}$ such that $1_{G} \notin \Gamma$ and $\widehat{G}=\Gamma \cup \Gamma^{-1} \cup\left\{1_{G}\right\}$. Let $\tau=\sum_{\gamma \in \Gamma} \oplus \gamma$. Then $1_{G_{d}}$ is weakly contained in $\Gamma_{d}$ by Remark 5.1(i) and so $1_{G_{d}} \preceq \tau_{d}$. On the other hand, it is clear that $\tau$ does not contain $1_{G}$, and so, by Remark 5.1(ii), $\tau$ does not weakly contain $1_{G}$. In the language of this paper, $\tau$ is $H_{d}$-amenable but is not $H$-amenable.

Let $\{\pi, C\}$ be the representation of $Z_{2}$ defined by setting $\pi(0)=1$ and $\pi(1)=-1$. Then $\pi^{\otimes 2}$ is unitarily equivalent to $1_{G}$, hence $1_{G} \subset \sum_{n=1}^{\infty} \oplus\left(\pi^{\otimes n}\right)$ $=\tau_{\pi}$. It follows that $\left\{\tau_{\pi}, \mathscr{H}_{\tau_{\pi}}\right\}$ is $H$-amenable, however, the justification from Example 2.5 shows that $\pi$ is not $H$-amenable. This shows that Lemma 4.2 is false if, in its statement, we replace "amenable" by " $H$-amenable". If $\pi$ is $H$-amenable, then Proposition 2.6 implies that $B(\pi)$ has an identity. This example shows as well that $A(\pi)$ (and therefore $B(\pi)$ ) may have an identity for representations $\pi$ which are not $H$-amenable.

As a special case of Example 5.2, we obtain a more interesting example of a non- $H$-amenable representation $\pi$ for which $\left\{\tau_{\pi}, \mathscr{H}_{\tau_{\pi}}\right\}$ is $H$-amenable. It is less trivial than the above example in the sense that for each $n, \pi^{\otimes n}$ is not $H$-amenable. It therefore also shows that Proposition 2.4 cannot be extended to infinite sums. Further, it provides us with a concrete description of a representation which is $H_{d}$-amenable, but not $H$-amenable.

EXAMPLE 5.3. Let $\pi=\iota \mathrm{c}$ be the unitary representation of the circle group, $G=\mathrm{T}$, as defined in Example 2.5, and consider $\tau_{\pi}$. Note that if we identify $Z$ with the dual group of $G$, then the representation $\pi^{\otimes n}$ is (up to unitary equivalence) just the character $\gamma_{n}$ on $G$ associated to the positive integer $n$. Observe that by choosing $s \in G$ for which $s^{n}=-1$, the argument from Example 2.5 shows that $\gamma_{n}$ is not $H_{d}$-amenable for any $n$. Now, by replacing $\Gamma$ in Example 5.2 with the set $\mathrm{N}$ of positive integers, we see that $\tau_{\pi}$ is just the representation $\tau$ from that example. Thus, $\tau=\tau_{\pi}$ is $H_{d}$-amenable (but not $H$-amenable). Moreover, note that $\tau$ is a representation on $l^{2}$ which affords the simple description $\tau(s)=\left(s^{n}\right)_{n} \in l^{\infty}$, where we are viewing $l^{\infty}$ as a $C^{*}$-subalgebra of $\mathscr{B}\left(l^{2}\right)$.

Assume now that $\Gamma$ is a locally compact abelian group with dual group $G$ (thus, $\widehat{\Gamma}=G$ and $\widehat{G}=\Gamma$ ). Recall that the Fourier-Stieltjes transform $\mu \mapsto \widehat{\mu}$ is an isometric $*$-isomorphism of $M(G)$ onto $B(\Gamma)$. Moreover, the FourierStieltjes transform is a $w^{*}-w^{*}$ homeomorphism. To see this, note that by Example 3 (1.17) of [9], the Fourier transform $L^{1}(\Gamma) \rightarrow C_{0}(G)$ extends to an isometric isomorphism, $\Lambda$, of $C^{*}(\Gamma)$ onto $C_{0}(G)$. A calculation shows that the Fourier-Stieltjes transform $M(G) \rightarrow B(\Gamma)$ is exactly the dual map $\Lambda^{*}$.

The measure algebra $M(G)$ becomes a Banach $C B(G)$-module through the 
operation defined by

$$
d(\phi \cdot \mu)(x)=\phi(x) d \mu(x) \quad(\phi \in C B(G), \quad \mu \in M(G)) .
$$

(By restricting this module operation to $C_{0}(G)$, we obtain the natural dual module action of $C_{0}(G)$ on $M(G)=C_{0}(G)^{*}$.) If $\gamma_{0} \in \Gamma$ is viewed, through duality, as an element of $C B(G)$, then for any $\mu \in M(G)$ we have

$$
\left(\widehat{\gamma_{0} \cdot \mu}\right)(\gamma)=\widehat{\mu}\left(\gamma-\gamma_{0}\right) \quad(\gamma \in \Gamma) .
$$

It follows that the Fourier-Stieltjes transform $A=\widehat{M}$ of a closed $\Gamma$-invariant subspace $M$ of $M(G)$ is a closed translation-invariant subspace of $B(\Gamma)$.

Let us also note that

$$
A(\gamma \otimes \bar{\gamma})=\overline{\langle u \bar{v}: u, v \in A(\gamma)\rangle}\|\cdot\| .
$$

Indeed, the statement follows from the calculation

$$
\left(\xi_{1} \otimes \eta_{1}\right) *_{\gamma \otimes \bar{\gamma}}\left(\xi_{2} \otimes \eta_{2}\right)=\left(\xi_{1} *_{\gamma} \xi_{2}\right) \overline{\left(\eta_{1} *_{\gamma} \eta_{2}\right)} .
$$

The following is an example of an $H$-amenable representation $\pi$ for which $A(\pi)=A_{\pi}$ does not have an approximate identity (let alone a bai). As we have already stated, we still do not know whether amenability (or $H$-amenability) of a representation $\gamma$ implies the existence of a bai in $A(\gamma \otimes \bar{\gamma})$. (In the example below we shall see that $\pi$ is not quasi-equivalent to a representation of the form $\gamma \otimes \bar{\gamma}$, and also that $1_{G} \in A(\pi \otimes \bar{\pi})$.)

Example 5.4. Let $G=(\mathrm{R},+)$, and let $S=((0, \infty),+)$. Then $S$ is a subsemigroup of $G$, so the semigroup algebra

$$
l^{1}(S)=\left\{\mu=\sum_{x>0} \mu(x) \delta_{x}: \sum_{x>0}|\mu(x)|<\infty\right\}
$$

is a closed subalgebra of $M(G)$. For $\gamma \in \Gamma$ and $\mu=\sum \mu(x) \delta_{x} \in l^{1}(S)$, we have $\gamma \cdot \mu=\sum \mu(x) \gamma(x) \delta_{x}$ which is also in $l^{1}(S)$. From the comments preceding this example, $A=\left\{\widehat{\mu}: \mu \in l^{1}(S)\right\}$ is a translation-invariant closed subalgebra of $B(\Gamma)(=B(\mathrm{R}))$, and by [1, Théorème 3.17], $A=A_{\pi}=A(\pi)$ for some representation $\pi$ of $\Gamma$. Let $x_{n}=\frac{1}{n}$ and consider $\delta_{x_{n}} \in l^{1}(S)$. Then for any $h \in C_{0}(G),\left\langle h, \delta_{x_{n}}\right\rangle=h\left(x_{n}\right) \rightarrow h(0)=\left\langle h, \delta_{0}\right\rangle$. Thus $\delta_{x_{n}} \rightarrow$ $\delta_{0} \sigma\left(M(G), C_{0}(G)\right)$ and it follows that $\widehat{\delta_{x_{n}}} \rightarrow \widehat{\delta_{0}}=1_{\Gamma} \sigma\left(B(\Gamma), C^{*}(\Gamma)\right)$. Consequently, $1_{\Gamma} \in \overline{A_{\pi}} w^{*}=B_{\pi}$ and $\pi$ is $H$-amenable. Now let $\mu=\sum_{x>0} \mu(x) \delta_{x}$ $\in l^{1}(S)$ and take $y \in S$. Then $\left(\delta_{y} * \mu\right)(y)=\left(\sum_{x>0} \mu(x) \delta_{y+x}\right)(y)=0$, so

$$
\left\|\delta_{y} * \mu-\delta_{y}\right\| \geq\left|\left(\delta_{y} * \mu\right)(y)-\delta_{y}(y)\right|=1 .
$$


But $\mu \in l^{1}(S)$ was arbitrary so $l^{1}(S)$, and therefore $A(\pi)$, does not have an approximate identity.

Note that $l^{1}(S)$ is not a $*$-subalgebra of $M(G)$, so $A(\pi)$ is not conjugation invariant; by the observation (7), $\pi$ is not quasi-equivalent to a representation of the form $\gamma \otimes \bar{\gamma}$. Finally note that by the same observation, taking any $x \in S$ we have $1_{\Gamma}=\widehat{\delta_{0}}=\left(\delta_{x} * \delta_{x}^{*}\right)^{-}=\widehat{\delta_{x}}\left(\widehat{\delta_{x}}\right)^{-} \in A(\pi \otimes \bar{\pi})$.

The Fourier algebra $A(G)=A_{\lambda}$ is an ideal in $B_{\lambda}$. With our final example, we observe that this is not always the case.

EXAMPLE 5.5. Let $\Gamma$ be any non-compact locally compact abelian group with dual group $G$. Then $G$ is non-discrete so $l^{1}(G)$ is a closed subalgebra of $M(G)$ which is not an ideal in $M(G)$. Clearly $l^{1}(G)$ is $\Gamma$-invariant, so $A=l^{1}(G)$ is of the form $A=A_{\pi}=A(\pi)$ for some representation $\pi$ of $G$. As well, $A(\pi)$ is not an ideal in $B(\Gamma)=\widehat{M(G)}$. But the Fourier-Stieltjes transform is a $w^{*}-w^{*}$ homeomorphism and $l^{1}(G)$ is $w^{*}$-dense in $M(G)$ so $B(\pi)=\overline{A(\pi)}{ }^{w^{*}}=B(\Gamma)$.

\section{Final Remarks}

The preprint [19] is, to some extent, a continuation of the work presented here. In it, we provide a class of examples for which the converse to Theorem 4.4 holds, and we study our Fourier algebras as operator Banach algebras, (especially with regards to operator amenability). Moreover, we define $C^{*}(\pi)=C_{\tau_{\pi}}^{*}, V N(\pi)=V N_{\tau_{\pi}}$, and respectively study the amenability and Connes-amenability of these algebras. When $\pi$ and $\bar{\pi}$ are weakly equivalent, we show that the nuclearity of $C^{*}(\pi)$ reflects the amenability of $G$ as well as we could wish: If $N$ is the kernel of $\pi$ in $G$, then $G / N$ is amenable if and only if $C^{*}(\pi)$ is nuclear and $G / N$ is inner amenable. Several corollaries are obtained in which we pay special attention to the conjugation and quasi-regular representations of $G$.

\section{REFERENCES}

1. Arsac, G., Sur l'espace de Banach engendré par les coefficients d'une représentation unitaire, Publ. Dép. Math. (Lyon) 13 (1976), 1-101.

2. Bédos, E., On the $C^{*}$-algebra generated by the left regular representation of a locally compact group, Proc. Amer. Math. Soc. 120 (1994), 603-608.

3. Bekka, M. E. B., Amenable unitary representations of locally compact groups, Invent. Math. 100 (1990), 383-401.

4. Bekka, M. E. B., Lau, A. T., and Schlichting, G., On invariant subalgebras of the FourierStieltjes algebra of a locally compact group, Math. Ann. 294 (1992), 513-522.

5. Bekka, B., de la Harpe, P., and Valette, A., Khazdan's property (T), manuscript. 
6. Belanger, A., and Forrest, B., Geometric properties of coefficient function spaces determined by unitary representations of a locally compact group, J. Math. Anal. Appl. 193 (1995), 390-405.

7. Derighetti, A., On the property of $P_{1}$ of locally compact groups, Comment. Math. Helv. 46 (1971), 226-239.

8. Dixmier, J., $C^{*}$-algebras (translated from the French), North-Holland, 1977.

9. Eymard, P., L'algébre de Fourier d'un groupe localement compact, Bull. Soc. Math. France 92 (1964), 181-236.

10. Granirer, E. E., and Leinert, M., On some topologies which coincide on the unit sphere of the Fourier-Stieltjes algebra B(G), Rocky Mountain J. Math. 11 (1981), 459-472.

11. Hewitt, E., and Ross, K. A., Abstract Harmonic Analysis, Volumes I and II, Springer-Verlag, New York, 1963 and 1970.

12. Johnson, B. E., Cohomology in Banach Algebras, Mem. Amer. Math. Soc. 127 (1972).

13. Kaniuth, E., Lau, A. T., and Schlichting, G., Lebesgue type decomposition of subspaces of Fourier-Stieltjes algebras, Trans. Amer. Math. Soc. 355 (2003), no. 4, 1467-1490.

14. Lau, A. T.-M., Analysis on a class of Banach algebras with applications to harmonic analysis on locally compact groups and semigroups, Fund. Math. 118 (1983), 161-175.

15. Leptin, H., Sur l'algebre de Fourier d'un groupe localement compact, C. R. Acad. Sci. Paris Ser. A 266 (1968), 1180-1182.

16. Runde, V., Lectures on amenability, Lecture Notes in Math. 1774 (2002).

17. Smith, R. R., and Spronk, N., Representations of group algebras in spaces of completely bounded maps, Indiana Univ. Math. J. (to appear).

18. Stokke, R., Quasi-central bounded approximate identities in group algebras of locally compact groups, Illinois J. Math. 48 (2004), 151-170.

19. Stokke, R., Amenability of Banach and $C^{*}$-algebras associated to unitary representations of locally compact groups, in preparation.

20. Yuan, C. K., The existence of inner invariant means on $L^{\infty}(G)$, J. Math. Anal. Appl. 130 (1988), 514-524.

DEPARTMENT OF MATHEMATICS AND STATISTICS

UNIVERSITY OF WINNIPEG

515 PORTAGE AVENUE

WINNIPEG

CANADA, R3B 2E9

E-mail: r.stokke@uwinnipeg.ca 